\title{
The New Kumaraswamy Kumaraswamy Family of Generalized Distributions with Application
}

\author{
Mahmoud R. Mahmoud \\ Institute of Statistical Studies \& Research \\ Cairo University, Egypt \\ El-Sayed A. El-Sherpieny \\ Institute of Statistical Studies \& Research \\ Cairo University, Egypt \\ Mohamed A. Ahmed \\ Institute of Statistical Studies \& Research \\ Cairo University, Egypt \\ mrmohamedali2005@yahoo.com
}

\begin{abstract}
Finding the best fitted distribution for data set becomes practically an important problem in world of data sets so that it is useful to use new families of distributions to fit more cases or get better fits than before. In this paper, a new generating family of generalized distributions so called the Kumaraswamy Kumaraswamy (KW-KW) family is presented. Four important common families of distributions are illustrated as special cases from the KW KW family. Moments, probability weighted moments, moment generating function, quantile function, median, mean deviation, order statistics and moments of order statistics are obtained. Parameters estimation and variance covariance matrix are computed using maximum likelihood method. A real data set is used to illustrate the potentiality of the KW KW weibull distribution (which derived from the $\mathrm{kw} \mathrm{kw}$ family) compared with other distributions.
\end{abstract}

Keywords: Kumaraswamy Kumaraswamy Distribution, Moments, Order Statistics, quantile function, Maximum Likelihood Estimation.

\section{Introduction}

The main idea of this paper is based on generating new families of generalized distributions, see Wahed (2006), to derive more generalized distributions from the following integration

$$
F(x ; T, W)=\int_{0}^{G_{1}(x ; W)} g_{2}(\mathrm{t} ; \mathrm{T}) d t ; 0<t<1 ;-\infty<x<\infty
$$

Where $G_{1}(x ; W)$ and $g_{1}(x ; W)$ are the cdf and pdf of the baseline distribution, $G_{2}(t ; T)$ and $g_{2}(t ; T)$ are the cdf and the pdf of the generator distribution, $\mathrm{T}$ is the parameters vector of the generator distribution and $\mathrm{W}$ is the parameters vector of the baseline distribution.

The contributions of this paper are four parts. First, we present the pdf and cdf of the new KW KW family of generalized distributions (section 2). Second, we calculate some properties of KW KW family like Moments, probability weighted moments, moment generating function, quantile function, median, mean deviation, order statistics and moments of order statistics (section 3). Third, we estimate KW KW family parameters 
using the maximum likelihood method and calculate the variance covariance matrix (section 4). Finally, we present a numerical example using real data on the KW KW Weibull and it gives the best fit between other distributions (section 5).

\section{The New Kumaraswamy Kumaraswamy Family}

El-Sherpieny and Ahmed (2014) presented the KW KW distribution where the cumulative distribution function (cdf) and the probability density function (pdf) respectively are:

$$
P(t ; a, b, \alpha, \beta)=1-\left\{1-\left[1-\left(1-t^{\alpha}\right)^{\beta}\right]^{a}\right\}^{b} ; 0<t<1 ; a, b, \alpha, \beta>0
$$

and

$$
p(t ; a, b, \alpha, \beta)=a b \alpha \beta t^{\alpha-1}\left(1-t^{\alpha}\right)^{\beta-1}\left[1-\left(1-t^{\alpha}\right)^{\beta}\right]^{a-1}\left\{1-\left[1-\left(1-t^{\alpha}\right)^{\beta}\right]^{a}\right\}^{b-1} ; 0<t<1 ; a, b, \alpha, \beta>0
$$

We will consider $p(t ; a, b, \alpha, \beta)$ as the pdf of the generator distribution $g_{2}(t ; T)$ in equation (1), then the cdf of the KW-KW family can be given by substituting equation (2) into equation (1) as follows:

$$
F(x ; T, W)=a b \alpha \beta \int_{0}^{G_{1}(x ; W)} t^{\alpha-1}\left(1-t^{\alpha}\right)^{\beta-1}\left[1-\left(1-t^{\alpha}\right)^{\beta}\right]^{a-1}\left\{1-\left[1-\left(1-t^{\alpha}\right)^{\beta}\right]^{a}\right\}^{b-1} d t
$$

Where $T=a, b, \alpha, \beta$ is the parameters vector of the generator distribution, $\mathrm{W}$ is the parameters vector of the baseline distribution and $G_{1}(x ; W)$ is the cdf of the baseline distribution.

Then,

$$
F(x ; T, W)=1-\left\{1-\left[1-\left(1-G_{1}^{\alpha}(x ; W)\right)^{\beta}\right]^{a}\right\}^{b} ;-\infty<x<\infty ; a, b, \alpha, \beta>0
$$

Differentiating equation (3) yields the pdf of the family:

$$
\begin{aligned}
f(x ; T, W)= & a b \alpha \beta g_{1}(x ; W) G_{1}^{\alpha-1}(x ; W)\left(1-G_{1}^{\alpha}(x ; W)\right)^{\beta-1}\left[1-\left(1-G_{1}^{\alpha}(x ; W)\right)^{\beta}\right]^{a-1} \\
& \times\left\{1-\left[1-\left(1-G_{1}^{\alpha}(x ; W)\right)^{\beta}\right]^{a}\right\}^{b-1} ;-\infty<x<\infty ; a, b, \alpha, \beta>0
\end{aligned}
$$

where $a, b, \alpha$ and $\beta$ are five shape parameters

Note that: if $b=1$, then the family of $\mathrm{KW} \mathrm{KW}$ reduces to the family of exponentiated $\mathrm{KW}$ see Cordeiro et al. (2013a), if $\mathrm{a}=1$ and $\mathrm{b}=1$, then it reduces to the family of KW see Cordeiro and Castro (2010), if $b=1$ and $\alpha=1$, then it reduces to the family of Exponentiated Generalized see Cordeiro et al. (2013b) and if $\mathrm{a}=1, \mathrm{~b}=1$ and $\beta=1$, then it reduces to the exponentiated family. 
An expansion of the density function (for simplicity) will be obtained as follows: Using binomial expansion in equation (2), where $b$ is a real non integer, yields

$$
\begin{aligned}
f(x ; T, W) & =a b \alpha \beta \sum_{i=0}^{\infty}(-1)^{i}\left(\begin{array}{c}
b-1 \\
i
\end{array}\right) g_{1}(x ; W) G_{1}^{\alpha-1}(x ; W)\left(1-G_{1}^{\alpha}(x ; W)\right)^{\beta-1} \\
& \times\left[1-\left(1-G_{1}^{\alpha}(x ; W)\right)^{\beta}\right]
\end{aligned}
$$

Then, using binomial expansion again in the last equation, where $a$ is real non integer, leads to:

$f(x ; T, W)=a b \alpha \beta \sum_{i, j=0}^{\infty}(-1)^{i+j}\left(\begin{array}{c}b-1 \\ i\end{array}\right)\left(\begin{array}{c}a(i+1)-1 \\ j\end{array}\right) g_{1}(x ; W) G_{1}^{\alpha-1}(x ; W)\left(1-G_{1}^{\alpha}(x ; W)\right)^{\beta(j+1)-1}$

and using binomial expansion more time in the last equation, where $\beta$ is real non integer, yields:

$f(x ; T, W)=a b \alpha \beta \sum_{i, j, k=0}^{\infty}(-1)^{i+j+k}\left(\begin{array}{c}b-1 \\ i\end{array}\right)\left(\begin{array}{c}a(i+1)-1 \\ j\end{array}\right)\left(\begin{array}{c}\beta(j+1)-1 \\ k\end{array}\right) g_{1}(x ; W) G_{1}{ }^{\alpha(k+1)-1}(x ; W)$ when $a, b$ and $\beta$ are integers $i$ stops at $b-1, j$ stops at $a(j+1)-1$ and $k$ stops at $\beta(j+1)-1$

Condition for the pdf expansion:

Since,

$a b \alpha \beta \sum_{i, j, k=0}^{\infty}(-1)^{i+j+k}\left(\begin{array}{c}b-1 \\ i\end{array}\right)\left(\begin{array}{c}a(i+1)-1 \\ j\end{array}\right)\left(\begin{array}{c}\beta(j+1)-1 \\ k\end{array}\right) \int_{-\infty}^{\infty} g_{1}(x ; W) G_{1}^{\alpha(k+1)-1}(x ; W) d x=1$

then,

$a b \alpha \beta \sum_{i, j, k=0}^{\infty}(-1)^{i+j+k}\left(\begin{array}{c}b-1 \\ i\end{array}\right)\left(\begin{array}{c}a(i+1)-1 \\ j\end{array}\right)\left(\begin{array}{c}\beta(j+1)-1 \\ k\end{array}\right)\left[\frac{G_{1}^{\alpha(k+1)}}{\alpha(k+1)}\right]_{-\infty}^{\infty}=1$

Hence,

$$
\frac{a b \beta}{k+1} \sum_{i, j, k=0}^{\infty}(-1)^{i+j+k}\left(\begin{array}{c}
b-1 \\
i
\end{array}\right)\left(\begin{array}{c}
a(i+1)-1 \\
j
\end{array}\right)\left(\begin{array}{c}
\beta(j+1)-1 \\
k
\end{array}\right)=1
$$

So the form of the expansion of the pdf with its condition is

$$
f(x ; T, W)=\sum_{i, j, k=0}^{\infty} w_{i, j, k} g_{1}(x ; W) G_{1}^{\alpha(k+1)-1}(x ; W)
$$

Where,

$$
w_{i, j, k}=a b \alpha \beta(-1)^{i+j+k}\left(\begin{array}{c}
b-1 \\
i
\end{array}\right)\left(\begin{array}{c}
a(i+1)-1 \\
j
\end{array}\right)\left(\begin{array}{c}
\beta(j+1)-1 \\
k
\end{array}\right)
$$

and

$$
\frac{1}{\alpha(k+1)} \sum_{i, j, k=0}^{\infty} w_{i, j, k}=1
$$


Another form can be yielded when $\alpha$ is real non integer by adding and subtracting 1 to $G_{1}^{\alpha(k+1)-1}(x, W)$ into equation (3) as follows

$f(x ; T, W)=a b \alpha \beta \sum_{i, j, k=0}^{\infty}(-1)^{i+j+k}\left(\begin{array}{c}b-1 \\ i\end{array}\right)\left(\begin{array}{c}a(i+1)-1 \\ j\end{array}\right)\left(\begin{array}{c}\beta(j+1)-1 \\ k\end{array}\right) g_{1}(x ; W)\left[1-\left(1-G_{1}(x ; W)\right)\right]^{\alpha(k+1)-1}$

Using binomial expansion yields:

$$
\begin{aligned}
f(x ; T, W) & =a b \alpha \beta \sum_{i, j, k, l=0}^{\infty}(-1)^{i+j+k+l}\left(\begin{array}{c}
b-1 \\
i
\end{array}\right)\left(\begin{array}{c}
a(i+1)-1 \\
j
\end{array}\right)\left(\begin{array}{c}
\beta(j+1)-1 \\
k
\end{array}\right)\left(\begin{array}{c}
\alpha(k+1)-1 \\
l
\end{array}\right) \\
& \times g_{1}(x ; W)\left(1-G_{1}(x ; W)\right)^{l}
\end{aligned}
$$

Using binomial expansion more time yields:

$$
\begin{aligned}
f(x ; T, W) & =a b \alpha \beta \sum_{i, j, k, l=0}^{\infty}(-1)^{i+j+k+l} \sum_{m=0}^{l}\left(\begin{array}{c}
b-1 \\
i
\end{array}\right)\left(\begin{array}{c}
a(i+1)-1 \\
j
\end{array}\right)\left(\begin{array}{c}
\beta(j+1)-1 \\
k
\end{array}\right)\left(\begin{array}{c}
\alpha(k+1)-1 \\
l
\end{array}\right)\left(\begin{array}{l}
l \\
m
\end{array}\right) \\
& \times g_{1}(x ; W) G_{1}^{m}(x ; W)
\end{aligned}
$$

Condition for the pdf expansion:

Since,

$$
\begin{aligned}
& a b \alpha \beta \sum_{i, j, k, l=0}^{\infty}(-1)^{i+j+k+l} \sum_{m=0}^{l}\left(\begin{array}{c}
b-1 \\
i
\end{array}\right)\left(\begin{array}{c}
a(i+1)-1 \\
j
\end{array}\right)\left(\begin{array}{c}
\beta(j+1)-1 \\
k
\end{array}\right)\left(\begin{array}{c}
\alpha(k+1)-1 \\
l
\end{array}\right)\left(\begin{array}{l}
l \\
m
\end{array}\right) \\
& \times \int_{=\infty}^{\infty} g_{1}(x ; W) G_{1}^{m}(x ; W) d x=1
\end{aligned}
$$

Then,

$$
a b \alpha \beta \sum_{i, j, k, l=0}^{\infty}(-1)^{i+j+k+l} \sum_{m=0}^{l}\left(\begin{array}{c}
b-1 \\
i
\end{array}\right)\left(\begin{array}{c}
a(i+1)-1 \\
j
\end{array}\right)\left(\begin{array}{c}
\beta(j+1)-1 \\
k
\end{array}\right)\left(\begin{array}{c}
\alpha(k+1)-1 \\
l
\end{array}\right)\left(\begin{array}{l}
l \\
m
\end{array}\right)\left[\frac{G_{1}^{m+1}}{m+1}\right]_{-\infty}^{\infty}=1
$$

Hence,

$$
\frac{a b \alpha \beta}{m+1} \sum_{i, j, k, l=0}^{\infty}(-1)^{i+j+k+l} \sum_{m=0}^{l}\left(\begin{array}{c}
b-1 \\
i
\end{array}\right)\left(\begin{array}{c}
a(i+1)-1 \\
j
\end{array}\right)\left(\begin{array}{c}
\beta(j+1)-1 \\
k
\end{array}\right)\left(\begin{array}{c}
\alpha(k+1)-1 \\
l
\end{array}\right)\left(\begin{array}{l}
l \\
m
\end{array}\right)=1
$$

So the another form of the expansion of the pdf with its condition is

$$
f(x ; T, W)=\sum_{i, j, k, l=0}^{\infty} \sum_{m=0}^{l} w_{i, j, k, l, m} g_{1}(x ; W) G_{1}^{m}(x ; W)
$$

Where,

$$
w_{i, j, k, l, m}=a b \alpha \beta(-1)^{i+j+k+l}\left(\begin{array}{c}
b-1 \\
i
\end{array}\right)\left(\begin{array}{c}
a(i+1)-1 \\
j
\end{array}\right)\left(\begin{array}{c}
\beta(j+1)-1 \\
k
\end{array}\right)\left(\begin{array}{c}
\alpha(k+1)-1 \\
l
\end{array}\right)\left(\begin{array}{c}
l \\
m
\end{array}\right)
$$


and

$\frac{1}{m+1} \sum_{i, j, k, l=0}^{\infty} \sum_{m=0}^{l} w_{i, j, k, l, m}=1$

An expansion for the cumulative function:

Using binomial expansion for $[F(x)]^{h}$, where $\mathrm{h}$ is integer, leads to:

Since,

$$
[F(x)]^{h}=\left\langle 1-\left\{1-\left[1-\left(1-G_{1}^{\alpha}(x ; W)\right)^{\beta}\right]^{a}\right\}^{b}\right\rangle^{h}
$$

Then,

$$
[F(x)]^{h}=\sum_{g=0}^{h}\left(\begin{array}{l}
h \\
g
\end{array}\right)(-1)^{g}\left\{1-\left[1-\left(1-G_{1}^{\alpha}(x, W)\right)^{\beta}\right]^{a}\right\}^{b g}
$$

Using binomial expansion another time, where $\mathrm{b}$ is real non integer, leads to

$$
[F(x)]^{h}=\sum_{g=0}^{h} \sum_{p=0}^{\infty}\left(\begin{array}{c}
h \\
g
\end{array}\right)\left(\begin{array}{c}
b g \\
p
\end{array}\right)(-1)^{g+p}\left[1-\left(1-G_{1}^{\alpha}(x ; W)\right)^{\beta}\right]^{a p}
$$

Using binomial expansion again, where $a$ is real non integer, yields

$$
[F(x)]^{h}=\sum_{g=0}^{h} \sum_{p, q=0}^{\infty}\left(\begin{array}{c}
h \\
g
\end{array}\right)\left(\begin{array}{c}
b \\
g \\
p
\end{array}\right)\left(\begin{array}{c}
a p \\
q
\end{array}\right)(-1)^{g+p+q}\left(1-G_{1}^{\alpha}(x ; W)\right)^{\beta q}
$$

Using binomial expansion again, where $\beta$ is real non integer, leads to

$$
[F(x)]^{h}=\sum_{g=0}^{h} \sum_{p, q, t=0}^{\infty}\left(\begin{array}{c}
h \\
g
\end{array}\right)\left(\begin{array}{c}
b g \\
p
\end{array}\right)\left(\begin{array}{c}
a p \\
q
\end{array}\right)\left(\begin{array}{c}
\beta q \\
t
\end{array}\right)(-1)^{g+p+q+t} G_{1}^{\alpha t}(x ; W)
$$

Adding and subtracting 1 to $G_{1}(x, W)$ into last equation yields

$$
[F(x)]^{h}=\sum_{g=0}^{h} \sum_{p, q, t=0}^{\infty}\left(\begin{array}{c}
h \\
g
\end{array}\right)\left(\begin{array}{c}
b g \\
p
\end{array}\right)\left(\begin{array}{c}
a p \\
q
\end{array}\right)\left(\begin{array}{c}
\beta q \\
t
\end{array}\right)(-1)^{g+p+q+t}\left[1-\left(1-G_{1}(x ; W)\right)\right]^{\alpha t}
$$

Using binomial expansion again leads to

$$
[F(x)]^{h}=\sum_{g=0}^{h} \sum_{p, q, t, f=0}^{\infty}\left(\begin{array}{c}
h \\
g
\end{array}\right)\left(\begin{array}{c}
b \\
g \\
p
\end{array}\right)\left(\begin{array}{c}
a p \\
q
\end{array}\right)\left(\begin{array}{c}
\beta q \\
t
\end{array}\right)\left(\begin{array}{c}
\alpha t \\
f
\end{array}\right)(-1)^{g+p+q+t+f}\left(1-G_{1}(x ; W)\right)^{f}
$$

Using binomial expansion again, where $f$ integer leads to

$$
[F(x)]^{h}=\sum_{g=0}^{h} \sum_{p, q, t, f=0}^{\infty} \sum_{z}^{f}\left(\begin{array}{l}
h \\
g
\end{array}\right)\left(\begin{array}{c}
b \\
g \\
p
\end{array}\right)\left(\begin{array}{c}
a p \\
q
\end{array}\right)\left(\begin{array}{c}
\beta q \\
t
\end{array}\right)\left(\begin{array}{c}
\alpha t \\
f
\end{array}\right)\left(\begin{array}{l}
f \\
z
\end{array}\right)(-1)^{g+p+q+t+f+z} G_{1}^{z}(x ; W)
$$


Replacing $\sum_{f=0}^{\infty} \sum_{z=0}^{f}$ with $\sum_{z=0}^{\infty} \sum_{f=z}^{\infty}$ yielding

$[F(x)]^{h}=\sum_{g=0}^{h} \sum_{p, q, t=0}^{\infty} \sum_{f=z}^{\infty} \sum_{z=0}^{\infty}\left(\begin{array}{l}h \\ g\end{array}\right)\left(\begin{array}{c}b g \\ p\end{array}\right)\left(\begin{array}{c}a p \\ q\end{array}\right)\left(\begin{array}{c}\beta q \\ t\end{array}\right)\left(\begin{array}{c}\alpha t \\ f\end{array}\right)\left(\begin{array}{l}f \\ z\end{array}\right)(-1)^{g+p+q+t+f+z} G_{1}^{z}(x ; W)$

Finally,

$$
[F(x)]^{h}=\sum_{z=0}^{\infty} s_{z} G_{1}^{z}(x ; W)
$$

Where,

$$
s_{z}=\sum_{g=0}^{h} \sum_{p, q, t=0}^{\infty} \sum_{f=z}^{\infty}\left(\begin{array}{l}
h \\
g
\end{array}\right)\left(\begin{array}{c}
b g \\
p
\end{array}\right)\left(\begin{array}{c}
a p \\
q
\end{array}\right)\left(\begin{array}{c}
\beta q \\
t
\end{array}\right)\left(\begin{array}{c}
\alpha t \\
f
\end{array}\right)\left(\begin{array}{l}
f \\
z
\end{array}\right)(-1)^{g+p+q+t+f+z}
$$

\section{Some properties of the Kumaraswamy Kumaraswamy Family}

In this section some properties of the $\mathrm{kw}-\mathrm{kW}$ family for any $\mathrm{KW} \mathrm{KW}$ generalized distribution will be obtained as follows:

\subsection{Moments}

If $\mathrm{X}$ has the pdf (6), then its $\mathrm{r}^{\text {th }}$ moments can be given from

$$
E\left(x^{r}\right)=\int_{-\infty}^{\infty} x^{r} f(x) d x
$$

First, when $\alpha$ is an integer, substituting equation (5) into equation (8) yields:

$$
E\left(x^{r}\right)=\sum_{i, j, k=0}^{\infty} w_{i, j, k} \int_{-\infty}^{\infty} x^{r} g_{(x ; w)} G_{1}^{\alpha(k+1)-1}(x ; W) d x
$$

Then,

$$
E\left(x^{r}\right)=\sum_{i, j, k=}^{\infty} w_{i, j, k} \tau_{r, \alpha(k+1)-1,0}
$$

Where, $\tau$ is the probability weighted moments (PWM) of the baseline distribution.

Second, when $\alpha$ is a real non integer, substituting equation (6) into equation (8) yields:

$$
E\left(x^{r}\right)=\sum_{i, j, k, l=0}^{\infty} \sum_{m=0}^{l} w_{i, j, k, l, m} \int_{-\infty}^{\infty} x^{r} g_{(x ; w)} G_{1}^{m}(x ; W) d x
$$

Then,

$$
E\left(x^{r}\right)=\sum_{i, j, k, l=0}^{\infty} \sum_{m=0}^{l} w_{i, j, k, l, m} \tau_{r, m, 0}
$$

Another form Based on the parent quantile function:

let,

$G(x ; w)=u$ 
First, when $\alpha$ is an integer yields

$E\left(x^{r}\right)=\sum_{i, j, k=0}^{\infty} w_{i, j, k} \int_{0}^{1}\left(Q_{G}(\mathrm{u})\right)^{r} u^{\alpha(k+1)-1} d u$

Second, when $\alpha$ is a real non integer yields

$E\left(x^{r}\right)=\sum_{i, j, k, l=0}^{\infty} \sum_{m=0}^{l} w_{i, j, k, l, m} \int_{0}^{1}\left(Q_{G}(\mathrm{u})\right)^{r} u^{m} d u$

\subsection{The Probability Weighted Moments}

The probability weighted moments (PWM) can be given from

$\tau_{s, r, 0}=E\left\{x^{s} F^{r}(x)\right\}$

Then,

$$
\tau_{s, r}=\int_{-\infty}^{\infty} x^{s} f(x) F^{r}(x) d x
$$

First when $\alpha$ is an integer

Substituting equation (5) \& (7) into equation (9), Replacing h with r, leads to:

$\tau_{s, r, 0}=\sum_{i, j, k=0}^{\infty} \sum_{z=0}^{\infty} s_{z} w_{i, j, k} \int_{-\infty}^{\infty} x^{s} g_{1}(x ; W) G_{1}^{z}(x ; W) d x$

Then,

$\tau_{s, r, 0}=\sum_{i, j, k=0}^{\infty} \sum_{z=0}^{\infty} s_{z} w_{i, j, k} \tau_{s, \alpha(k+1)+z, 0}$

Second, when $\alpha$ is a real non integer, Substituting equation (6) \& (7) into equation (9), Replacing h with r, leads to:

$\tau_{s, r, 0}=\sum_{i, j, k, l=0}^{\infty} \sum_{m=0}^{l} \sum_{z=0}^{\infty} s_{z} w_{i, j, k} \int_{-\infty}^{\infty} x^{s} g_{1}(x ; W) G_{1}^{m+z}(x ; W) d x$

Then,

$\tau_{s, r, 0}=\sum_{i, j, k, l=0}^{\infty} \sum_{m=0}^{l} \sum_{z=0}^{\infty} s_{z} w_{i, j, k} \tau_{s, m+z, 0}$

Another form Based on the parent quantile function:

First, when $\alpha$ is integer yields

$\tau_{s, r, 0}=\sum_{i, j, k=0}^{\infty} \sum_{z=0}^{\infty} s_{z} w_{i, j, k} \int_{0}^{1}\left(Q_{G}(\mathrm{u})\right)^{s} u^{z} d u$ 
Second, when $\alpha$ is real non integer yields

$$
\tau_{s, r, 0}=\sum_{i, j, k, l=0}^{\infty} \sum_{m=0}^{l} \sum_{z=0}^{\infty} s_{z} w_{i, j, k} \int_{0}^{1}\left(\mathrm{Q}_{\mathrm{G}}(\mathrm{u})\right)^{s} u^{m+z} d u
$$

\subsection{The Moment Generating Function}

Generally, the moment generating function is:

$$
M_{x}(t)=E\left(e^{t x}\right)
$$

and using expansion at the last equation leads to

$$
M_{x}(t)=E\left(\sum_{r=0}^{\infty} \frac{x^{r} t^{r}}{r !}\right)
$$

So,

$$
M_{x}(t)=\sum_{r=0}^{\infty} \frac{E\left(x^{r}\right) t^{r}}{r !}
$$

Hence,

$$
M_{x}(t)=E\left(x^{r}\right) \sum_{r=0}^{\infty} \frac{t^{r}}{r !}
$$

Another form Based on the parent quantile function:

First, when $\alpha$ is integer

$$
M_{u}(t)=\sum_{i, j, k=0}^{\infty} w_{i, j, k} \int_{0}^{1} \exp \left(\mathrm{tQ}_{\mathrm{G}}(\mathrm{u})\right) u^{\alpha(k+1)-1} d u
$$

Second, when $\alpha$ is real non integer

$$
M_{u}(t)=\sum_{i, j, k, l=0}^{\infty} \sum_{m=0}^{l} w_{i, j, k, l, m} \int_{0}^{1} \exp \left(\mathrm{tQ}_{\mathrm{G}}(\mathrm{u})\right) u^{m} d u
$$

\subsection{Quantile Function and The Median}

The quantile function of the KW KW family can be gotten from the cdf of the KW KW family.

As follows:

Let, $u=1-\left\{1-\left[1-\left(1-G_{1}^{\alpha}(x ; W)\right)^{\beta}\right]^{a}\right\}^{b} ; 0<u<1, x \in R$

Then,

$Q_{G}\left[1-\left[1-\left[1-(1-u)^{\frac{1}{b}}\right]^{\frac{1}{a}}\right]^{\frac{1}{\beta}}\right]^{\frac{1}{\alpha}}=x$

Since, median can be yielded by substituting u with 0.5 
Hence,

Median $=Q_{G}\left[1-\left[1-\left[1-\left(\frac{1}{2}\right)^{\frac{1}{b}}\right]^{\frac{1}{a}}\right]^{\frac{1}{\beta}}\right]^{\frac{1}{\alpha}}$

\subsection{The Mean Deviation}

Basically, the mean deviation is a measure for the amount of scatter in X.

Generally, the mean deviation is expressed by:

$\delta_{1}(x)=\int_{-\infty}^{\infty}|x-\mu| f(x) d x \quad$ and $\quad \delta_{2}(x)=\int_{-\infty}^{\infty}|x-M| f(x) d x$

where, $\mu$ is mean and $\mathrm{M}$ is median.

These measures can be expressed easily as:

$\delta_{1}(X)=2 \mu F(\mu)-2 T(\mu) \quad$ and $\quad \delta_{2}(X)=\mu-2 T(M)$

where,

$T(q)=\int_{-\infty}^{q} x f(x) d x$

Which is the first incomplete moment

Another form Based on the parent quantile function:

First, when $\alpha$ is integer yields

$T(q)=\sum_{i, j, k=0}^{\infty} w_{i, j, k} \int_{0}^{\mathrm{G}(\mathrm{q})} Q_{G}(\mathrm{u}) u^{\alpha(k+1)-1} d u$

Second, when $\alpha$ is real non integer yields

$T(q)=\sum_{i, j, k, l=0}^{\infty} \sum_{m=0}^{l} w_{i, j, k, l, m} \int_{0}^{G(\mathrm{q})} Q_{G}(\mathrm{u}) u^{m} d u$

\subsection{Order Statistics}

The pdf of the $\mathrm{u}^{\text {th }}$ order statistics can be given from, see Arnold et al. (1992)

$$
f_{(u: n)}\left(x_{u: n}\right)=\frac{f\left(x_{u}\right)}{B(u, n-u+1)} F^{u-1}\left(x_{u}\right)\left\{1-F\left(x_{u}\right)\right\}^{n-u}
$$

Then, we can use binomial expansion into $F^{u-1}\left(x_{u}\right)\left\{1-F\left(x_{u}\right)\right\}^{n-u}$ as follows $F^{u-1}\left(x_{u}\right)\left\{1-F\left(x_{u}\right)\right\}^{n-u}=\sum_{w=0}^{n-u}\left(\begin{array}{c}n-u \\ w\end{array}\right)(-1)^{w} F^{u+w-1}(x)$ 
Substituting equation (7) into last equation, Replacing $u+w-1$ with $r$, leads to:

$$
F^{u-1}\left(x_{u}\right)\left\{1-F\left(x_{u}\right)\right\}^{n-u}=\sum_{w=0}^{n-u}\left(\begin{array}{c}
n-u \\
w
\end{array}\right)(-1)^{w} \sum_{z=0}^{\infty} s_{z} G_{1}^{z}(x ; W)
$$

Then,

$$
F^{u-1}\left(x_{u}\right)\left\{1-F\left(x_{u}\right)\right\}^{n-u}=\sum_{w=0}^{n-u} p_{z, w} G_{1}^{z}(x ; W)
$$

Where,

$$
p_{z, w}=\sum_{z=0}^{\infty} s_{z}\left(\begin{array}{c}
n-u \\
w
\end{array}\right)(-1)^{w}
$$

First: when $\alpha$ is integer

Substituting equation (5) and (11) into (10) leads to

$$
f_{(u: n)}\left(x_{u: n}\right)=\frac{g_{1}\left(x_{u} ; W\right)}{B(u, n-u+1)} \sum_{i, j, k=0}^{\infty} \sum_{w=0}^{n-u} w_{i, j, k} p_{z, w} G_{1}^{z+\alpha(k+1)-1}\left(x_{u} ; W\right)
$$

Second, when $\alpha$ is real non integer

Substituting (6) and (11) into (10) leads to

$f_{(u: n)}\left(x_{u: n}\right)=\frac{g_{1}\left(x_{u} ; W\right)}{B(u, n-u+1)} \sum_{i, j, k, l, m=0}^{\infty} \sum_{m=0}^{l} \sum_{w=0}^{n-u} w_{i, j, k, l, m} p_{z, w} G_{1}^{m+z}\left(x_{u} ; W\right)$

\section{Moments of Order Statistics}

Moments of order statistics is defined by

$$
E_{(u: n)}\left(x_{u}^{r}\right)=\int_{-\infty}^{\infty} x_{u}^{r} f_{(u: n)}\left(x_{u}\right) d x_{u}
$$

First, when $\alpha$ is integer, substituting equation (12) into equation (14) leads to

$$
E_{(u: n)}\left(x_{u}^{r}\right)=\frac{1}{B(u, n-u+1)} \sum_{i, j, k=0}^{\infty} \sum_{w=0}^{n-u} p_{z, w} w_{i, j, k} \int_{-\infty}^{\infty} x_{u}^{r} g_{1}\left(x_{u} ; W\right) G_{1}^{z+\alpha(k+1)-1}\left(x_{u} ; W\right) d x_{u}
$$

Then,

$$
E_{(u: n)}\left(x_{u}^{r}\right)=\frac{1}{B(u, n-u+1)} \sum_{i, j, k=0}^{\infty} \sum_{w=0}^{n-u} p_{z, w} w_{i, j, k} \tau_{r, z+\alpha(k+1)-1,0}
$$

Second, when $\alpha$ is real non integer, substituting equation (13) into equation (14) leads to $E_{(u: n)}\left(x_{u}^{r}\right)=\frac{1}{B(u, n-u+1)} \sum_{i, j, k, l, m=0}^{\infty} \sum_{m=0}^{l} \sum_{w=0}^{n-u} w_{i, j, k, l, m} p_{z, w} \int_{-\infty}^{\infty} x_{u}^{r} g_{1}\left(x_{u} ; W\right) G_{1}^{m+z}\left(x_{u} ; W\right) d x_{u}$

Then,

$$
E_{(u: n)}\left(x_{u}^{r}\right)=\frac{1}{B(u, n-u+1)} \sum_{i, j, k, l, m=0}^{\infty} \sum_{m=0}^{l} \sum_{w=0}^{n-u} w_{i, j, k, l, m} p_{z, w} \tau_{r, m+z}
$$


Since the L-moments are linear functions of expected order statistics, see Hosking (1990):

$$
\lambda_{s+1}=(s+1)^{-1} \sum_{y=0}^{s}(-1)^{y}\left(\begin{array}{l}
s \\
y
\end{array}\right) E\left(x_{s+1-y: s+1}\right), s=0,1, \ldots
$$

So the first four L-moments can be calculated from

$$
\begin{aligned}
& \lambda_{1}=E\left(X_{1: 1}\right) \\
& \lambda_{2}=\frac{1}{2} E\left(X_{2: 2}-2 X_{1: 2}\right) \\
& \lambda_{3}=\frac{1}{3} E\left(X_{3: 3}-2 X_{2: 3}+X_{1: 3}\right) \\
& \lambda_{4}=\frac{1}{4} E\left(X_{4: 4}-3 X_{3: 4}+3 X_{2: 4}-X_{1: 4}\right)
\end{aligned}
$$

\section{Maximum Likelihood Estimators}

let $\mathrm{X}_{1}, \mathrm{X}_{2} \ldots \mathrm{Xn}$ be the iid random variables from the $\mathrm{KW}-\mathrm{KW}(a, \mathrm{~b}, \alpha, \beta)$ distribution and $T=a, b, \alpha, \beta$ is the parameters vector of the generator distribution and $\mathrm{W}$ is the parameters vector of the baseline distribution, then The likelihood function is

$$
\begin{aligned}
L(x ; T, W)= & (a b \alpha \beta)^{n} \prod_{i=1}^{n}\left\{1-\left[1-\left(1-G_{1}^{\alpha}\left(x_{i} ; W\right)\right)^{\beta}\right]^{a}\right\}^{b-1}\left[1-\left(1-G_{1}^{\alpha}\left(x_{i} ; W\right)\right)^{\beta}\right]^{a-1} \\
& \times \prod_{i=0}^{n}\left(1-G_{1}^{\alpha}\left(x_{i} ; W\right)\right)^{\beta-1} G_{1}^{\alpha-1}\left(x_{i} ; W\right) g_{1}\left(x_{i} ; W\right)
\end{aligned}
$$

and the log likelihood is

$$
\begin{aligned}
l(x ; T, W)= & n[\log a+\log b+\log \alpha+\log \beta]+(b-1) \sum_{i=1}^{n} \log \left\{1-\left[1-\left(1-G_{1}{ }^{\alpha}\left(x_{i} ; W\right)\right)^{\beta}\right]^{a}\right\} \\
& +(a-1) \sum_{i=1}^{n} \log \left[1-\left(1-G_{1}^{\alpha}\left(x_{i} ; W\right)\right)^{\beta}\right]+(\beta-1) \sum_{i=1}^{n} \log \left(1-G_{1}^{\alpha}\left(x_{i} ; W\right)\right) \\
& +(\alpha-1) \sum_{i=1}^{n} \log G_{1}\left(x_{i} ; W\right)+\sum_{i=1}^{n} \log g_{1}\left(x_{i} ; W\right)
\end{aligned}
$$

Partial differentiating with respect to $a$ yields:

$$
\begin{aligned}
\frac{\partial l(x ; T, W)}{\partial a}= & \frac{n}{a}-(b-1) \sum_{i=1}^{n} \frac{\left[1-\left(1-G_{1}^{\alpha}\left(x_{i} ; W\right)\right)^{\beta}\right]^{a} \log \left[1-\left(1-G_{1}^{\alpha}\left(x_{i} ; W\right)\right)^{\beta}\right]}{\left\{1-\left[1-\left(1-G_{1}^{\alpha}\left(x_{i}, W\right)\right)^{\beta}\right]^{a}\right\}} \\
& +\sum_{i=1}^{n} \log \left[1-\left(1-G_{1}^{\alpha}\left(x_{i} ; W\right)\right)^{\beta}\right]
\end{aligned}
$$


Then.

$\frac{\partial l(x ; T, W)}{\partial a}=\frac{n}{a}+\sum_{i=1}^{n} \log \left[1-\left(1-G_{1}^{\alpha}\left(x_{i} ; W\right)\right)^{\beta}\right]\left\{1-(b-1) \sum_{i=1}^{n} \frac{\left[1-\left(1-G_{1}^{\alpha}\left(x_{i} ; W\right)\right)^{\beta}\right]^{a}}{\left\{1-\left[1-\left(1-G_{1}{ }^{\alpha}\left(x_{i} ; W\right)\right)^{\beta}\right]^{a}\right\}}\right\}$

Partial differentiating with respect to $b$ yields:

$\frac{\partial l(x ; T, W)}{\partial b}=\frac{n}{b}+\sum_{i=1}^{n} \log \left\{1-\left[1-\left(1-G_{1}{ }^{\alpha}\left(x_{i} ; W\right)\right)^{\beta}\right]^{a}\right\}$

Partial differentiating with respect to $\alpha$ yields:

$$
\begin{aligned}
& \frac{\partial l(x ; T, W)}{\partial \alpha}=\frac{n}{\alpha}-a \beta(b-1) \sum_{i=1}^{n} \frac{\left[1-\left(1-G_{1}^{\alpha}\left(x_{i} ; W\right)\right)^{\beta}\right]^{a-1}}{\left\{1-\left[1-\left(1-G_{1}^{\alpha}\left(x_{i} ; W\right)\right)^{\beta}\right]^{a}\right\}}\left(1-G_{1}^{\alpha}\left(x_{i} ; W\right)\right)^{\beta-1} \\
& \quad \times G_{1}^{\alpha}\left(x_{i} ; W\right) \log G_{1}\left(x_{i} ; W\right)+\beta(a-1) \sum_{i=1}^{n} \frac{\left(1-G_{1}^{\alpha}\left(x_{i} ; W\right)\right)^{\beta-1}}{1-\left(1-G_{1}^{\alpha}\left(x_{i} ; W\right)\right)^{\beta}} G_{1}^{\alpha}\left(x_{i} ; W\right) \log G_{1}\left(x_{i} ; W\right) \\
& -(\beta-1) \sum_{i=1}^{n} \frac{G_{1}^{\alpha}\left(x_{i} ; W\right) \log G_{1}\left(x_{i} ; W\right)}{\left(1-G_{1}^{\alpha}\left(x_{i} ; W\right)\right)}+\sum_{i=1}^{n} \log G_{1}\left(x_{i} ; W\right)
\end{aligned}
$$

then,

$$
\begin{aligned}
\frac{\partial l(x ; T, W)}{\partial \alpha}= & \frac{n}{\alpha}+\sum_{i=1}^{n}\left(1-G_{1}^{\alpha}\left(x_{i} ; W\right)\right)^{\beta-1} G_{1}^{\alpha}\left(x_{i} ; W\right) \log G_{1}\left(x_{i} ; W\right)\left\{\frac{\beta(a-1)}{1-\left(1-G_{1}^{\alpha}\left(x_{i} ; W\right)\right)^{\beta}}\right. \\
& -\frac{a \beta(b-1)\left[1-\left(1-G_{1}^{\alpha}\left(x_{i} ; W\right)\right)^{\beta}\right]^{a-1}}{\left\{1-\left[1-\left(1-G_{1}^{\alpha}\left(x_{i} ; W\right)\right)^{\beta}\right]^{\alpha}\right\}}-\frac{(\beta-1)}{\left.\left(1-G_{1}^{\alpha}\left(x_{i} ; W\right)\right)^{\beta}\right\}}+\sum_{i=1}^{n} G_{1}\left(x_{i} ; W\right)
\end{aligned}
$$

Partial differentiating with respect to $\beta$ yields:

$$
\begin{gathered}
\frac{\partial l(x ; T, W)}{\partial \beta}=\frac{n}{\beta}+a(b-1) \sum_{i=1}^{n} \frac{\left[1-\left(1-G_{1}^{\alpha}\left(x_{i} ; W\right)\right)^{\beta}\right]^{a-1}}{\left\{1-\left[1-\left(1-G_{1}^{\alpha}\left(x_{i} ; W\right)\right)^{\beta}\right]^{a}\right\}}\left(1-G_{1}^{\alpha}\left(x_{i} ; W\right)\right)^{\beta} \log \left(1-G_{1}^{\alpha}\left(x_{i} ; W\right)\right) \\
-(a-1) \sum_{i=1}^{n} \frac{\left(1-G_{1}^{\alpha}\left(x_{i} ; W\right)\right)^{\beta}}{1-\left(1-G_{1}^{\alpha}\left(x_{i} ; W\right)\right)^{\beta}} \log \left(1-G_{1}^{\alpha}\left(x_{i} ; W\right)\right)+\sum_{i=1}^{n} \log \left(1-G_{1}^{\alpha}\left(x_{i} ; W\right)\right)
\end{gathered}
$$


Then,

$$
\begin{aligned}
\frac{\partial l(x ; T, W)}{\partial \beta} & =\frac{n}{\beta}+\sum_{i=1}^{n}\left(1-G_{1}^{\alpha}\left(x_{i} ; W\right)\right)^{\beta} \log \left(1-G_{1}^{\alpha}\left(x_{i} ; W\right)\left\{\frac{a(b-1)\left[1-\left(1-G_{1}^{\alpha}\left(x_{i} ; W\right)\right)^{\beta}\right]^{a-1}}{\left\{1-\left[1-\left(1-G_{1}^{\alpha}\left(x_{i} ; W\right)\right)^{\beta}\right]^{a}\right\}}\right.\right. \\
& \left.-\frac{(a-1)}{1-\left(1-G_{1}^{\alpha}\left(x_{i} ; W\right)\right)^{\beta}}+\frac{1}{\left(1-G_{1}^{\alpha}\left(x_{i} ; W\right)\right)^{\beta}}\right\}
\end{aligned}
$$

Partial differentiating with respect to $W_{\mathrm{i}}$ yields:

$$
\begin{aligned}
& \frac{\partial l(x ; T, W)}{\partial W_{j}}=-\alpha \beta a(b-1) \sum_{i=1}^{n} \frac{\left[1-\left(1-G_{1}^{\alpha}\left(x_{i} ; W\right)\right)^{\beta}\right]^{\alpha-1}}{\left\{1-\left[1-\left(1-G_{1}^{\alpha}\left(x_{i} ; W\right)\right)^{\beta}\right]^{a}\right\}}\left(1-G_{1}^{\alpha}\left(x_{i} ; W\right)\right)^{\beta-1} G_{1}^{\alpha-1}\left(x_{i} ; W\right) \frac{\partial G_{1}\left(x_{i} ; W\right)}{\partial w_{j}} \\
& +\alpha \beta(a-1) \sum_{i=1}^{n} \frac{\left(1-G_{1}^{\alpha}\left(x_{i} ; W\right)\right)^{\beta-1}}{1-\left(1-G_{1}^{\alpha}\left(x_{i} ; W\right)\right)^{\beta}} G_{1}^{\alpha-1}\left(x_{i} ; W\right) \frac{\partial G_{1}\left(x_{i} ; W\right)}{\partial w_{j}} \\
& \quad-\alpha(\beta-1) \sum_{i=1}^{n} \frac{G_{1}^{\alpha-1}\left(x_{i} ; W\right)}{1-G_{1}^{\alpha}\left(x_{i} ; W\right)} \frac{\partial G_{1}\left(x_{i} ; W\right)}{\partial w_{j}}+(\alpha-1) \sum_{i=1}^{n} \frac{1}{G_{1}\left(x_{i} ; W\right)} \frac{\partial G_{1}\left(x_{i} ; W\right)}{\partial w_{j}} \\
& \quad+\sum_{i=1}^{n} \frac{1}{g_{1}\left(x_{i} ; W\right)} \frac{\partial g_{1}\left(x_{i} ; W\right)}{\partial w_{j}}
\end{aligned}
$$

Then,

$$
\begin{aligned}
\frac{\partial l(x ; T, W)}{\partial W_{j}} & =\sum_{i=1}^{n} \frac{G_{1}^{\alpha-1}\left(x_{i} ; W\right)}{G_{1}\left(x_{i} ; W\right)} \frac{\partial G_{1}\left(x_{i} ; W\right)}{\partial w_{j}}\left\{(\alpha-1) G_{1}^{1-\alpha}\left(x_{i} ; W\right)\right. \\
& -\alpha \beta a(b-1) \sum_{i=1}^{n} \frac{\left[1-\left(1-G_{1}^{\alpha}\left(x_{i} ; W\right)\right)^{\beta}\right]^{\alpha-1}}{\left\{1-\left[1-\left(1-G_{1}^{\alpha}\left(x_{i} ; W\right)\right)^{\beta}\right]^{a}\right\}}\left(1-G_{1}^{\alpha}\left(x_{i} ; W\right)\right)^{\beta-1} G_{1}\left(x_{i} ; W\right) \\
& \left.+\alpha \beta(a-1) \sum_{i=1}^{n} \frac{\left(1-G_{1}^{\alpha}\left(x_{i} ; W\right)\right)^{\beta-1}}{1-\left(1-G_{1}^{\alpha}\left(x_{i} ; W\right)\right)^{\beta}} G_{1}\left(x_{i} ; W\right)-\alpha(\beta-1) \sum_{i=1}^{n} \frac{G_{1}\left(x_{i} ; W\right)}{1-G_{1}^{\alpha}\left(x_{i} ; W\right)}\right\} \\
& +\sum_{i=1}^{n} \frac{1}{g_{1}\left(x_{i} ; W\right)} \frac{\partial g_{1}\left(x_{i} ; W\right)}{\partial w_{j}}
\end{aligned}
$$


Equating these partial derivatives to zero, yields a system of non linear equations that needs to be solved numerically to obtain parameters estimation, Numerical maximization of the log-likelihood above is accomplished by using the RS method see Rigby and Stasinopoulos (2005).

Let $\theta$ is the vector of the unknown parameters $\left(a, b, \alpha, \beta, w_{j}\right)$, then the element of the $5 \times 5$ information matrix $\mathrm{I}\left(a, b, \alpha, \beta, w_{j}\right)$ can be approximated by:

$I_{i j}(\hat{\theta})=E\left[-\left.\frac{\partial^{2} \ell(\theta)}{\partial \theta_{i} \partial \theta_{j}}\right|_{\theta=\hat{\theta}}\right]$

$I^{1}\left(a, b, \alpha, \beta, w_{j}\right)$ is the variance covariance matrix of the unknown parameters $\left(a, b, \alpha, \beta, w_{j}\right)$ and the asymptotic distributions of the MLE parameters are

$$
\sqrt{n}\left(\hat{\theta}_{i}-\theta_{i}\right) \approx N_{5}\left(0, I^{-1}\left(\hat{\theta}_{i}\right)\right), i=1, \ldots ., 5
$$

The approximation $100(1-\alpha) \%$ confidence intervals for the unknown parameters, based on the asymptotic distributions of the $\mathrm{KW}-\mathrm{KW}$ family distributions, are determined, respectively, as

$$
\hat{\theta}_{i} \pm z_{\frac{\alpha}{2}} \sqrt{I^{-1}\left(\hat{\theta}_{i}\right)}, i=1, \ldots ., 5
$$

where, $z_{\frac{\alpha}{2}}$ is the upper $\frac{\alpha}{2}$ th percentile of a standard normal distribution.

\section{Application}

In this section we give a real data to illustrate an example for one distribution of the new family of KW-KW distributions so called the the Kumaraswamy - Kumaraswamy Weibull distribution to see how the new model works practically and we will use the Mathematica package version 10 to do that. In our example, We used different distributions like the kumaraswamy - kumaraswamy - Weibull (KW-KW-W) distribution [derived from the KW KW family], the exponentiated kumaraswamy weibull (E-KW-W) distribution [derived from the $\mathrm{E} \mathrm{KW}$ family], the kumaraswamy weibull $(\mathrm{KW}-\mathrm{W})$ distribution [derived from the KW family], the exponentiated generalized weibull (EG$\mathrm{W})$ distribution [derived from the $\mathrm{E} G$ family], the exponentiated weibull (E-W) distribution [derived from the E family] and the weibull (W) distribution.

The following data represents the strengths of $1.5 \mathrm{~cm}$ glass fibres for 27 devices, originally obtained by workers at the UK National Physical Laboratory. Unfortunately, the units of measurement are not given in the paper, see http://www.npl.co.uk/.

$0.17,0.13,0.16,0.14,0.20,0.15,0.13,0.11,0.15,0.12,0.12,0.15,0.12,0.16,0.21,0.20$, $0.23,0.16,0.12,0.10,0.32,0.33,0.33,0.36,0.38,0.20,0.26$ 


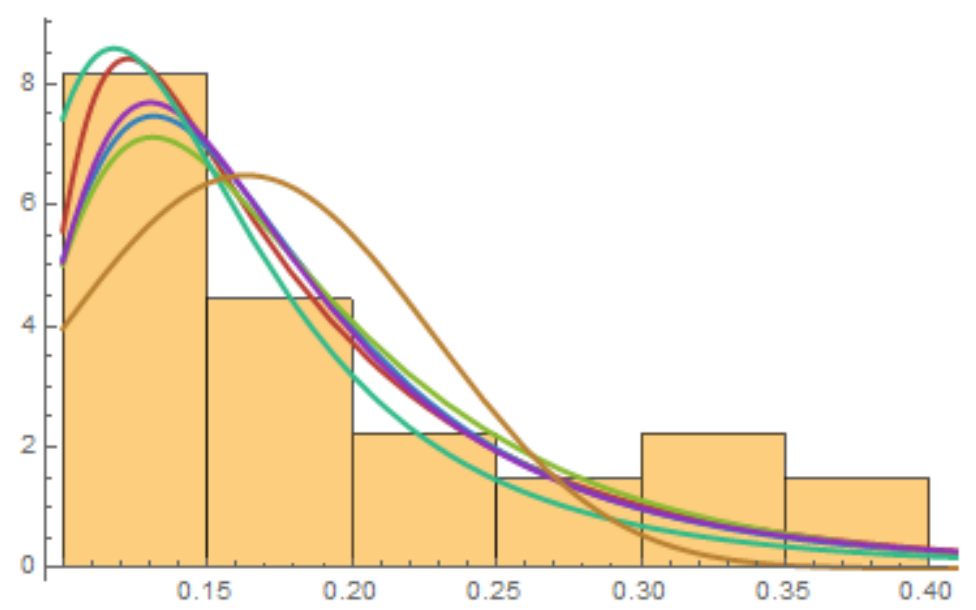

- KW-KW-Weibul

- E-KW-Weibull

- KW-Weibull

- EG-Weibull

- E-Weibull

- Weibull

Figure 1: Probability density functions for different distributions

Table 1: The MLE of the parameter(s) and the associated AIC and BIC values

\begin{tabular}{|l|l|l|l|l|l|}
\hline The Model & MLE of parameters & K.S & p-value & AIC & BIC \\
\hline KW - KW - W $(a, b, \alpha, \beta, \theta, \lambda)$ & $(26.99,0.17,13,3.58,0.77,45.11)$ & 0.161 & 0.381 & -74.026 & -65.2193 \\
\hline E - KW - W $(a, \alpha, \beta, \theta, \lambda)$ & $(152.84,4.89,4.91,0.28,172.13)$ & 0.168 & 0.182 & -69.1345 & -61.5859 \\
\hline KW - W $(\alpha, \beta, \theta, \lambda)$ & $(37.75,0.35,1.09,29.49)$ & 0.17 & 0.191 & -67.986 & -61.6956 \\
\hline EG - W $(a, \beta, \theta, \lambda)$ & $(168336,10.74,0.238,11.374)$ & 0.159 & 0.124 & -63.419 & -50.4372 \\
\hline E - W $(\alpha, \theta, \lambda)$ & $(20880.9,0.29,21634.8)$ & 0.2 & 0.095 & -70.6536 & -63.6212 \\
\hline $\mathrm{W}(\theta, \lambda)$ & $(3.08,5.39)$ & 0.227 & 0.054 & -51.7742 & -47.9999 \\
\hline
\end{tabular}

In Table (1) we compute the MLE of distributions parameters, Kolmogorov-Smirnov (K.S) test statistic, AIC and BIC for every distribution. We find from K.S test statistic that at level of significance 0.05 we can not reject that the data fits all earlier distributions but it fits more the KW - KW - W ( $a, b, \alpha, \beta, \theta, \lambda)$ distribution. We see that the KW - KW - W (a, b, $\alpha, \beta, \theta, \lambda)$ distribution has the smallest AIC and BIC so the KW - KW - W (a, b, $\alpha, \beta, \theta, \lambda)$ distribution can be the best fitted distribution compared with earlier distributions

Table 2: The log-likelihood function, The likelihood ratio test statistic and p-values

\begin{tabular}{|l|l|l|l|l|l|}
\hline The Model & $\mathrm{H}_{0}$ & $\begin{array}{l}\ell \\
(\log \text { likelihood) }\end{array}$ & $\begin{array}{l}\Lambda \\
(\text { The likelihood } \\
\text { ratio test statistic) }\end{array}$ & $\begin{array}{l}\mathrm{df} \\
\text { (degrees of } \\
\text { freedom) }\end{array}$ & P-value \\
\hline $\mathrm{E}-\mathrm{KW}-\mathrm{W}(\mathrm{a}, \alpha, \beta, \theta, \lambda)$ & $\mathrm{b}=0$ & 31.5136 & 2.921 & 1 & 0.0238 \\
\hline $\mathrm{KW}-\mathrm{W}(\alpha, \beta, \theta, \lambda)$ & $\mathrm{a}=0, \mathrm{~b}=0$ & 31.0791 & 2.869 & 2 & 0.0871 \\
\hline $\mathrm{EG}-\mathrm{W}(\mathrm{a}, \beta, \theta, \lambda)$ & $\alpha=0, \mathrm{~b}=0$ & 30.5947 & 3.589 & 2 & 0.00514 \\
\hline $\mathrm{E}-\mathrm{W}(\alpha, \theta, \lambda)$ & $\mathrm{a}=0, \mathrm{~b}=0, \beta=0$ & 29.1843 & 6.6586 & 3 & 0.00842 \\
\hline $\mathrm{W}(\theta, \lambda)$ & $\mathrm{a}=0, \mathrm{~b}=0, \alpha=0, \beta=0$ & 10.4911 & 44.045 & 4 & $6.279 \times 10^{-9}$ \\
\hline
\end{tabular}


In Table (2) and based on the likelihood ratio test, where the KW- KW-W (a, b, $\alpha, \beta, \theta, \lambda)$ distribution generalizes the E-KW-W $(\mathrm{a}, \alpha, \beta, \theta, \lambda)$ distribution, the $\mathrm{KW}-\mathrm{W}(\alpha, \beta, \theta, \lambda)$ distribution, the $\mathrm{EG}-\mathrm{W}(\mathrm{a}, \beta, \theta, \lambda)$ distribution, the $\mathrm{E}-\mathrm{W}(\alpha, \theta, \lambda)$ distribution, the $\mathrm{E}-\mathrm{W}$ $(\alpha, \theta, \lambda)$ distribution and the $\mathrm{W}(\theta, \lambda)$ distribution, we find from the p-values that we can reject all null hypotheses when the level of significance is 0.1 .

\section{Conclusions}

The new Kumaraswamy Kumaraswamy family of generalized distributions can be useful in world of data sets because of its flexible properties and its generalization of some important families of distributions like the families of Exponentiated Kumaraswamy, Kumaraswamy, Exponentiated Generalized and the Exponentiated. It is clear from the application that the new model so called the Kumaraswamy Kumaraswamy Weibull distribution which was derived from the Kumaraswamy Kumaraswamy family practically gave the best fit compared with other distributions. We encourage researchers to do more researches and applications on other distributions of the Kumaraswamy Kumaraswamy family in univariate and multivariate cases.

\section{Appendix}

The elements of the observed information matrix $\mathrm{I}\left(a, b, \alpha, \beta, w_{j}\right)$ for the parameters are:

Let:

$$
\begin{aligned}
& A_{i}=\left\{1-\left[1-\left(1-G_{1}^{\alpha}\left(x_{i} ; \mathrm{w}\right)\right)^{\beta}\right]^{\alpha}\right\}^{2}, B_{i}=\left[1-\left(1-G_{1}^{\alpha}\left(x_{i} ; \mathrm{w}\right)\right)^{\beta}\right]^{\alpha}, C_{i}=\left(1-G_{1}^{\alpha}\left(x_{i} ; \mathrm{w}\right)\right)^{\beta}, \\
& D_{i}=G_{1}^{\alpha}\left(x_{i} ; \mathrm{w}\right), E_{i}=G_{1}\left(x_{i}, \mathrm{w}\right) \text { and } H_{i}=g_{1}\left(x_{i} ; \mathrm{w}\right)
\end{aligned}
$$

Then,

$$
\begin{aligned}
\frac{\partial^{2} l(x ; T, W)}{\partial a^{2}}= & \frac{n}{a^{2}}-(b-1) \sum_{i=1}^{n}\left\{\frac{\left(1-B_{i}\right) \log \left(1-C_{i}\right) B_{i} \log \left(1-C_{i}\right)+B_{i} \log \left(1-C_{i}\right) B_{i} \log \left(1-C_{i}\right)}{A_{i}}\right\} \\
\frac{\partial^{2} l(x ; T, W)}{\partial a \partial b}= & -\sum_{i=1}^{n} \frac{\mathrm{B}_{i} \log \left(1-C_{i}\right)}{1-\mathrm{B}_{i}}, \\
\frac{\partial^{2} l(x ; T, W)}{\partial a \partial \alpha}= & -(b-1) \sum_{i=1}^{n}\left\{\frac{\left(1-B_{i}\right) B_{i} \frac{\beta\left(1-D_{i}\right)^{\beta-1} D_{i} \log E_{i}}{1-C_{i}}}{A_{i}}\right. \\
& +\frac{\left(1-B_{i}\right) \log \left(1-C_{i}\right) a\left(1-C_{i}\right)^{a-1} \beta\left(1-D_{i}\right)^{\beta-1} D_{i} \log E_{i}}{A_{i}} \\
& \left.+\frac{B_{i} \log \left(1-C_{i}\right) a\left(1-C_{i}\right)^{a-1} \beta\left(1-D_{i}\right)^{\beta-1} D_{i} \log E_{i}}{A_{i}}\right\} \\
& +\sum_{i=1}^{n} \frac{\beta\left(1-D_{i}\right)^{\beta-1} D_{i} \log E_{i}}{\left(1-C_{i}\right)}
\end{aligned}
$$




$$
\begin{aligned}
& \frac{\partial^{2} l(x ; T, W)}{\partial a \partial \beta}=-(b-1) \sum_{i=1}^{n}\left\{\frac{-\left(1-B_{i}\right) \log \left(1-C_{i}\right) a\left(1-C_{i}\right)^{a-1} C_{i} \log \left(1-D_{i}\right)}{A_{i}}\right. \\
& \left.-\frac{\left(1-B_{i}\right) B_{i} \frac{C_{i} \log \left(1-D_{i}\right)}{\left(1-C_{i}\right)}-B_{i} \log \left(1-C_{i}\right) a\left(1-B_{i}\right)^{a-1} C_{i} \log \left(1-D_{i}\right)}{A_{i}}\right\} \\
& -\sum_{i=1}^{n} \frac{C_{i} \log \left(1-D_{i}\right)}{\left(1-C_{i}\right)} \\
& \frac{\partial^{2} l(x ; T, W)}{\partial a \partial W_{j}}=-(b-1) \sum_{i=1}^{n}\left\{\frac{\left(1-B_{i}\right) B_{i} \frac{\beta\left(1-D_{i}\right)^{\beta-1} \alpha\left(E_{i}\right)^{\alpha-1} \frac{\partial E_{i}}{\partial W_{j}}}{\left(1-C_{i}\right)}}{A_{i}}\right. \\
& +\frac{\left(1-B_{i}\right)\left\{\log \left(1-C_{i}\right)\right\} a\left(1-C_{i}\right)^{a-1} \beta\left(1-D_{i}\right)^{\beta-1} \alpha\left(E_{i}\right)^{\alpha-1} \frac{\partial E_{i}}{\partial W_{j}}}{A_{i}} \\
& \left.+\frac{B_{i}\left\{\log \left(1-C_{i}\right)\right\} a\left(1-C_{i}\right)^{a-1} \beta\left(1-D_{i}\right)^{\beta-1} \alpha\left(E_{i}\right)^{\alpha-1} \frac{\partial E_{i}}{\partial W_{j}}}{A_{i}}\right\} \\
& +\sum_{i=1}^{n} \frac{\beta\left(1-D_{i}\right)^{\beta-1} \alpha\left(E_{i}\right)^{\alpha-1} \frac{\partial E_{i}}{\partial W_{j}}}{\left(1-C_{i}\right)} \\
& \frac{\partial^{2} l(x ; T, W)}{\partial b^{2}}=\frac{-n}{b^{2}}, \\
& \frac{\partial^{2} l(x ; T, W)}{\partial b \partial \alpha}=-\sum_{i=1}^{n}\left\{\frac{a\left(1-C_{i}\right)^{a-1} \beta\left(1-D_{i}\right)^{\beta-1} D_{i} \log E_{i}}{\left(1-B_{i}\right)}\right\} \text {, } \\
& \frac{\partial^{2} l(x ; T, W)}{\partial b \partial \beta}=\sum_{i=1}^{n}\left\{\frac{a\left(1-C_{i}\right)^{a-1} C_{i} \log \left(1-D_{i}\right)}{\left(1-B_{i}\right)}\right\}
\end{aligned}
$$




$$
\begin{aligned}
& \frac{\partial^{2} l(x ; T, W)}{\partial b \partial W_{i}}=-\sum_{i=1}^{n}\left\{\frac{a\left(1-C_{i}\right)^{a-1} \beta\left(1-D_{i}\right)^{\beta-1} \alpha\left(E_{i}\right)^{\alpha-1} \frac{\partial E_{i}}{\partial W_{j}}}{\left(1-B_{i}\right)}\right\} \\
& \frac{\partial^{2} l(x ; T, W)}{\partial \alpha \partial \alpha}=\frac{-n}{\alpha^{2}}-a \beta(b-1) \sum_{i=1}^{n}\left\{\frac{\left(1-C_{i}\right)^{a-1}}{\left(1-B_{i}\right)}\left(1-D_{i}\right)^{\beta-1} D_{i}\left[\log E_{i}\right]^{2}\right. \\
& -\frac{\left(1-C_{i}\right)^{a-1}}{\left(1-B_{i}\right)} D_{i}\left(\log E_{i}\right)(B-1)\left(1-D_{i}\right)^{\beta-2} D_{i}\left(\log E_{i}\right) \\
& +\left(1-D_{i}\right)^{\beta-1} D_{i}\left(\log E_{i}\right) \frac{\left(1-B_{i}\right)(a-1)\left(1-C_{i}\right)^{a-2} \beta\left(1-D_{i}\right)^{\beta-1} D_{i}\left(\log E_{i}\right)}{A_{i}} \\
& \left.+\frac{\left(1-C_{i}\right)^{a-1} a\left(1-C_{i}\right)^{a-1} \beta\left(1-D_{i}\right)^{\beta-1} D_{i}\left(\log E_{i}\right)}{A_{i}}\right\} \\
& +\beta(a-1) \sum_{i=1}^{n}\left\{\frac{\left(1-D_{i}\right)^{\beta-1}}{\left(1-C_{i}\right)} D_{i}\left[\log E_{i}\right]^{2}\right. \\
& \left.-D_{i} \log E_{i} \frac{\left(1-C_{i}\right)(\beta-1)\left(1-D_{i}\right)^{\beta-2} D_{i}\left(\log E_{i}\right)-\left(1-D_{i}\right)^{\beta-1} \beta\left(1-D_{i}\right)^{\beta-1} D_{i} \log E_{i}}{\left(1-C_{i}\right)^{2}}\right\} \\
& +(\beta-1) \sum_{i=1}^{n}\left\{\frac{\left(1-D_{i}\right)\left(\log E_{i}\right) D_{i}\left(\log E_{i}\right)+D_{i}\left(\log E_{i}\right) D_{i}\left(\log E_{i}\right)}{\left(1-D_{i}\right)^{2}}\right\}, \\
& \frac{\partial^{2} l(x ; T, W)}{\partial \alpha \partial \beta}=a(b-1) \sum_{i=1}^{n}\left\{\frac{\left(1-C_{i}\right)^{a-1}}{\left(1-B_{i}\right)}\left(1-D_{i}\right)^{\beta-1} D_{i}\left(\log E_{i}\right)\right. \\
& +a \beta(b-1)\left(1-D_{i}\right)^{\beta-1} \log \left(1-D_{i}\right) D_{i}\left(\log E_{i}\right) \frac{\left(1-C_{i}\right)^{a-1}}{\left(1-B_{i}\right)} \\
& +\left(1-D_{i}\right)^{\beta-1} D_{i}\left(\log E_{i}\right) \frac{\left(1-B_{i}\right)(a-1)\left(1-C_{i}\right)^{a-2} C_{i} \log \left(1-D_{i}\right)}{A_{i}} \\
& \left.-\frac{\left(1-C_{i}\right)^{a-1} a\left(1-C_{i}\right)^{a-1} C_{i} \log \left(1-D_{i}\right)}{A_{i}}\right\} \\
& +(a-1) \sum_{i=1}^{n} \frac{\left(1-D_{i}\right)^{\beta-1}}{\left(1-C_{i}\right)} D_{i}\left(\log E_{i}\right)+\beta(a-1) \sum_{i=1}^{n}\left\{D_{i}\left(\log E_{i}\right)\right. \\
& \left.\times \frac{\left(1-C_{i}\right)\left(1-D_{i}\right)^{\beta-1} \log \left(1-D_{i}\right)}{\left(1-C_{i}\right)^{2}}+\frac{\left(1-D_{i}\right)^{\beta-1}\left(1-D_{i}\right)^{\beta} \log \left(1-D_{i}\right)}{\left(1-C_{i}\right)^{2}}\right\} \\
& -\sum_{i=1}^{n} \frac{D_{i}\left(\log E_{i}\right)}{\left(1-D_{i}\right)}
\end{aligned}
$$




$$
\begin{aligned}
& \frac{\partial^{2} l(x ; T, W)}{\partial \beta \partial \beta}=\frac{-n}{\beta^{2}}+a(b-1) \sum_{i=1}^{n}\left\{C_{i}\left(\log \left(1-D_{i}\right)\right)^{2} \frac{\left(1-C_{i}\right)^{a-1}}{\left(1-B_{i}\right)}\right. \\
& -C_{i} \log \left(1-D_{i}\right) \frac{\left(1-B_{i}\right)(a-1)\left(1-C_{i}\right)^{a-2} C_{i}\left(\log \left(1-D_{i}\right)\right)}{A_{i}} \\
& \left.-\frac{\left(1-C_{i}\right)^{a-1} a\left(1-C_{i}\right)^{a-1} C_{\mathrm{i}} \log \left(1-D_{i}\right)}{A_{i}}\right\} \\
& -(a-1) \sum_{i=1}^{n}\left\{\log \left(1-D_{i}\right) \frac{\left(1-C_{i}\right) C_{i} \log \left(1-D_{i}\right)}{\left(1-C_{i}\right)^{2}}\right. \\
& +\frac{C_{i}^{2} \log \left(1-D_{i}\right)}{\left(1-C_{i}\right)^{2}}, \\
& \frac{\partial^{2} l(x ; T, W)}{\partial \alpha \partial w_{j}}=-a \beta(b-1) \sum_{i=1}^{n}\left\{\frac{\left(1-C_{i}\right)^{a-1}}{\left(1-B_{i}\right)}\left(1-D_{i}\right)^{\beta-1} \frac{D_{i}}{E_{i}} \frac{\partial E_{i}}{\partial w_{j}}\right. \\
& +\frac{\left(1-C_{i}\right)^{a-1}}{\left(1-B_{i}\right)}\left(1-D_{i}\right)^{\beta-1} \alpha E_{i}^{\alpha-1} \log E_{i} \frac{\partial E_{i}}{\partial w_{j}} \\
& -\frac{\left(1-C_{i}\right)^{a-1}}{\left(1-B_{i}\right)} D_{i} \log E_{i}(B-1)\left(1-D_{i}\right)^{\beta-2} \alpha E_{i}^{\alpha-1} \frac{\partial E_{i}}{\partial w_{j}} \\
& +\left(1-D_{i}\right)^{\beta-1} D_{i}\left(\log E_{i}\right) \frac{\left(1-B_{i}\right)(a-1)\left(1-C_{i}\right)^{a-2} \beta\left(1-D_{i}\right)^{\beta-1} \alpha E_{i}^{\alpha-1} \frac{\partial E_{i}}{\partial w_{j}}}{A_{i}} \\
& \left.+\frac{\left(1-C_{i}\right)^{a-1} a\left(1-C_{i}\right)^{a-1}+\beta\left(1-D_{i}\right)^{\beta-1} \alpha E_{i}^{\alpha-1} \frac{\partial E_{i}}{\partial w_{j}}}{A_{i}}\right\} \\
& +\beta(a-1) \sum_{i=1}^{n}\left\{\frac{\left(1-D_{i}\right)^{\beta-1}}{\left(1-C_{i}\right)} \frac{D_{i}}{E_{i}} \frac{\partial E_{i}}{\partial w_{j}}+\frac{\left(1-D_{i}\right)^{\beta-1}}{\left(1-C_{i}\right)}\left(\log E_{i}\right) \alpha E_{i}^{\alpha-1} \frac{\partial E_{i}}{\partial w_{j}}\right. \\
& +D_{i}\left(\log E_{i}\right) \frac{\left(1-C_{i}\right)(\beta-1)\left(1-D_{i}\right)^{\beta-2} \alpha E_{i}^{\alpha-1} \frac{\partial E_{i}}{\partial w_{j}}}{\left(1-C_{i}\right)^{2}} \\
& \left.-\frac{\left(1-D_{i}\right)^{\beta-1} \beta\left(1-D_{i}\right)^{\beta-1} \alpha E_{i}^{\alpha-1} \frac{\partial E_{i}}{\partial w_{j}}}{\left(1-C_{i}\right)^{2}}\right\}-(B-1) \sum_{i=1}^{n}\left\{\frac{D_{i}}{\left(1-D_{i}\right)} \frac{1}{E_{i}} \frac{\partial E_{i}}{\partial w_{j}}\right. \\
& \left.+\frac{\left(\log E_{i}\right) \alpha E_{i}^{\alpha-1}}{\left(1-D_{i}\right)} \frac{\partial E_{i}}{\partial w_{j}}+D_{i}\left(\log E_{i}\right) \frac{\alpha E_{i}^{\alpha-1} \frac{\partial E_{i}}{\partial w_{j}}}{\left(1-D_{i}\right)^{2}}\right\}+\sum_{i=1}^{n} \frac{1}{E_{i}} \frac{\partial E_{i}}{\partial w_{j}},
\end{aligned}
$$




$$
\begin{aligned}
& \frac{\partial^{2} l(x ; T, W)}{\partial \beta \partial W_{i}}=a(b-1) \sum_{i=1}^{n}\left\{-C_{i} \frac{\alpha E_{i}^{\alpha-1} \frac{\partial E_{i}}{\partial w_{j}}}{\left(1-D_{i}\right)} \frac{\left(1-C_{i}\right)^{a-1}}{\left(1-B_{i}\right)}\right. \\
& -\log \left(1-D_{i}\right) \beta\left(1-D_{i}\right)^{\beta-1} \alpha E_{i}^{\alpha-1} \frac{\partial E_{i}}{\partial w_{j}} \frac{\left(1-C_{i}\right)^{a-1}}{\left(1-B_{i}\right)} \\
& +C_{i} \log \left(1-D_{i}\right) \frac{\left(1-B_{i}\right)(a-1)\left(1-C_{i}\right)^{a-2} \beta\left(1-D_{i}\right)^{\beta-1} \alpha E_{i}{ }^{\alpha-1} \frac{\partial E_{i}}{\partial w_{j}}}{A_{i}} \\
& \left.+\frac{\left(1-C_{i}\right)^{a-1} a\left(1-C_{i}\right)^{a-1} \beta\left(1-D_{i}\right)^{\beta-1} \alpha E_{i}^{\alpha-1} \frac{\partial E_{i}}{\partial w_{j}}}{A_{i}}\right\} \\
& +(a-1) \sum_{i=1}^{n}\left\{\frac{C_{i} \alpha E_{i}^{\alpha-1} \frac{\partial E_{i}}{\partial w_{j}}}{\left(1-C_{i}\right)\left(1-D_{i}\right)}-\log \left(1-D_{i}\right) \frac{\left(1-C_{i}\right) \beta\left(1-D_{i}\right)^{\beta-1} \alpha E_{i}^{\alpha-1} \frac{\partial E_{i}}{\partial w_{j}}}{\left(1-C_{i}\right)^{2}}\right. \\
& \left.-\frac{C_{i} \beta\left(1-D_{i}\right)^{\beta-1} \alpha E_{i}^{\alpha-1} \frac{\partial E_{i}}{\partial w_{j}}}{\left(1-C_{i}\right)^{2}}\right\}-\sum_{i=1}^{n} \frac{\alpha E_{i}^{\alpha-1} \frac{\partial E_{i}}{\partial w_{j}}}{\left(1-D_{i}\right)},
\end{aligned}
$$

and 


$$
\begin{aligned}
& \frac{\partial^{2} l(x ; T, W)}{\partial W_{j} \partial W_{k}}=-\alpha \beta a(b-1) \sum_{i=1}^{n}\left\{\frac{\left(1-C_{i}\right)^{a-1}}{\left(1-B_{i}\right)}\left(1-D_{i}\right)^{\beta-1} E_{i}^{\alpha-1} \frac{\partial^{2} E_{i}}{\partial w_{j} \partial w_{k}}\right. \\
& +\frac{\left(1-C_{i}\right)^{a-1}}{\left(1-B_{i}\right)}\left(1-D_{i}\right)^{\beta-1}(\alpha-1) E_{i}^{\alpha-2} \frac{\partial E_{i}}{\partial w_{k}} \frac{\partial E_{i}}{\partial w_{j}} \\
& -\frac{\left(1-C_{i}\right)^{a-1}}{\left(1-B_{i}\right)} E_{i}^{\alpha-1} \frac{\partial E_{i}}{\partial w_{j}}(\beta-1)\left(1-D_{i}\right)^{\beta-2} \alpha E_{i}^{\alpha-1} \frac{\partial E_{i}}{\partial w_{k}} \\
& +\left(1-D_{i}\right)^{\beta-1} E_{i}^{\alpha-1} \frac{\partial E_{i}}{\partial w_{j}} \frac{\left(1-B_{i}\right)(a-1)\left(1-C_{i}\right)^{a-2} \beta\left(1-D_{i}\right)^{\beta-1} \alpha E_{i}^{\alpha-1} \frac{\partial E_{i}}{\partial w_{k}}}{A_{i}} \\
& \left.+\frac{\left(1-C_{i}\right)^{a-1} a\left(1-C_{i}\right)^{a-1} \beta\left(1-D_{i}\right)^{\beta-1} \alpha E_{i}^{\alpha-1} \frac{\partial E_{i}}{\partial w_{k}}}{A_{i}}\right\} \\
& +\alpha \beta(a-1) \sum_{i=1}^{n}\left\{\frac{\left(1-D_{i}\right)^{\beta-1}}{\left(1-C_{i}\right)} E_{i}^{\alpha-1} \frac{\partial^{2} E_{i}}{\partial w_{j} \partial w_{k}}+\frac{\left(1-D_{i}\right)^{\beta-1}}{\left(1-C_{i}\right)} \frac{\partial E_{i}}{\partial w_{j}}(\alpha-1) E_{i}^{\alpha-2} \frac{\partial E_{i}}{\partial w_{k}}\right. \\
& -E_{i}^{\alpha-1} \frac{\partial E_{i}}{\partial w_{j}} \frac{\left(1-C_{i}\right)(\beta-1)\left(1-D_{i}\right)^{\beta-2} \alpha E_{i}^{\alpha-1} \frac{\partial E_{i}}{\partial w_{k}}}{\left(1-C_{i}\right)^{2}} \\
& \left.-\frac{\left(1-D_{i}\right)^{\beta-1} \beta\left(1-D_{i}\right)^{\beta-1} \alpha E_{i}^{\alpha-1} \frac{\partial E_{i}}{\partial w_{k}}}{\left(1-C_{i}\right)^{2}}\right\} \\
& -\alpha(\beta-1) \sum_{i=1}^{n}\left\{\frac{E_{i}^{\alpha-1}}{\left(1-D_{i}\right)} \frac{\partial^{2} E_{i}}{\partial w_{j} \partial w_{k}}\right. \\
& \left.+\frac{\partial E_{i}}{\partial w_{j}} \frac{\left(1-D_{i}\right)(\alpha-1) E_{i}^{\alpha-2} \frac{\partial E_{i}}{\partial w_{k}}+E_{i}^{\alpha-1} \alpha E_{i}^{\alpha-1} \frac{\partial E_{i}}{\partial w_{k}}}{\left(1-D_{i}\right)^{2}}\right\} \\
& +(\alpha-1) \sum_{i=1}^{n}\left\{\frac{1}{E_{i}} \frac{\partial^{2} E_{i}}{\partial w_{j} \partial w_{k}}-\frac{\partial E_{i}}{\partial w_{j}} \frac{\frac{\partial E_{i}}{\partial w_{k}}}{E_{i}^{2}}\right\} \\
& +\sum_{i=1}^{n}\left\{\frac{1}{H_{i}} \frac{\partial^{2} H_{i}}{\partial w_{j} \partial w_{k}}-\frac{1}{H_{i}^{2}} \frac{\partial H_{i}}{\partial w_{j}} \frac{\partial H_{i}}{\partial w_{k}}\right\}
\end{aligned}
$$




\section{Acknowledgements}

We thank the referees for their helpful comments and anyone provided an improved suggestion for the paper.

\section{References}

1. A.S. Wahed, (2006). A general method of constructing extended families of distribution from an existing continuous class: Journal of Probability and Statistical Science. 4, 165-177.

2. C. B. Arnold, N. Balakrishnan, H. N. Nagaraja, (1992). A first course in order statistics: John Wiley \& Sons, Inc. New York.

3. E.A. El-Sherpieny, M. A. Ahmed, (2014). On the Kumaraswamy Kumaraswamy distribution: International Journal of Basic and Applied Sciences. 3, 372-381.

4. G. M. Cordeiro, A. J. lemonte, W. B. Souza, (2013a). The exponentiated Kumaraswamy distribution and its log-transform: Brazilian Journal of Probability and Statistics. 27, 31-53.

5. G. M. Cordeiro, M. Castro, (2010). A new family of generalized distributions: Journal of Statistical Computation \& Simulation. 81, 1563-5163.

6. G. M. Cordeiro, E.M.M. Ortega, D.C.C. Cunha, (2013b). The Exponentiated Generalized Class of Distributions: Journal of Data Science. 11, 1-27.

7. J. R. M. Hosking, (1990). L moments: analysis and estimation of distribution using linear combination of order statistic, Journal of the Royal Statistical Society. Series B, 52, 105-124.

8. R.A. Rigby and D.M. Stasinopoulos, (2005). Generalized additive models for location, scale and shape (with discussion): Appl. Stat. 54, 507-554.

9. The UK National Physical Laboratory, http://www.npl.co.uk/ last visit 10/1/2014. 